\section{Comorbilidad en personas con depresión que consultan en centros de la atención primaria de salud en Santiago, Chile}

PABLO MARTÍNEZ ${ }^{1,2,3, a}$, GRACIELA ROJAS ${ }^{1,2}$, ROSEMARIE FRITSCH $^{1}$, VANIA MARTÍNEZ ${ }^{2,4}$, PAUL A. VÖHRINGER ${ }^{1,2,5}$, ARIEL CASTRO ${ }^{6, b}$

\section{Comorbidity in people with depression seeking help at primary health care centers in Santiago, Chile}

Background: International evidence has shown the complex interaction between depression and chronic physical diseases. Depression in scenarios involving multiple comorbidities has not received enough attention in Chile. Aim: To characterize the depressed people who consult at Primary Health Care Centers (PHCCs), taking into account the presence of chronic physical or psychiatric comorbidity. Materials and Methods: A secondary analysis of databases used in a clinical trial. Two hundred fifty six adults seeking professional help were recruited in four PHCCs located in the Metropolitan Region. These people had a major depressive episode, identified with a structured psychiatric interview (MINI), and gave their informed consent to participate. Socio-demographic information was collected, depressive symptomatology was measured with the patient health questionnaire 9 (PHQ-9), psychiatric morbidity was assessed using the Mini International Neuropsychiatric Interview (MINI), and chronic physical diseases were self-reported by the patients. Descriptive analyses of all the variables were conducted. Results: Seventy percent of patients had a history of depression, with a median of two prior depressive episodes. Depressive symptoms were mostly considered as moderate to severe and severe and $31 \%$ of the patients had high suicide risk. Seventy eight percent displayed a physical or psychiatric comorbidity. Of these patients, $29 \%$ only had a chronic physical comorbidity, while $46 \%$ suffered from an additional psychiatric disorder. Conclusions: Depressed individuals who seek help at PHCCs constitute an especially complex population that must be treated taking into account multiple comorbidities.

(Rev Med Chile 2017; 145: 25-32)

Key words: Chronic Disease; Comorbidity; Depression; Depressive Disorder; Primary Health Care.
'Departamento de Psiquiatría y Salud Mental, Hospital Clínico Universidad de Chile, Santiago, Chile.

${ }^{2}$ Instituto Milenio para la Investigación en Depresión y Personalidad, Santiago, Chile. ${ }^{3}$ Centro de Innovación en Tecnologías de la Información para Aplicaciones Sociales CITIAPS, Universidad de Santiago de Chile, Santiago, Chile.

${ }^{4}$ Centro de Medicina

Reproductiva y Desarrollo Integral del Adolescente (CEMERA), Facultad de Medicina, Universidad de Chile, Santiago, Chile.

${ }^{5}$ Mood Disorders Program, Tufts Medical Center, Tufts University, Boston, USA.

${ }^{6}$ Subdirección de Investigación, Hospital Clínico Universidad de Chile. Santiago, Chile.

aPsicólogo, Alumno de Programa de Doctorado en Psicología, Universidad de Santiago de Chile. ${ }^{b} Q u i ́ m i c o$ Farmacéutico, Magíster en Epidemiología Clínica.

Este estudio fue financiado por el Fondo Nacional de Desarrollo Científico y Tecnológico (FONDECYT), a través del proyecto $\mathrm{N}^{\circ} 1130230$. FONDECYT no tuvo influencia en el diseño del estudio; tampoco en la recolección, análisis o interpretación de los datos; ni en la preparación, revisión o aprobación del manuscrito.

Recibido el 5 de agosto de 2016, aceptado el 26 de diciembre de 2016.

Correspondencia: Graciela Rojas Avenida La Paz 1003, Recoleta, Santiago, Chile. graciela.rojas.castillo@usach.cl
E $\mathrm{n}$ un escenario de disminución de las tasas de mortalidad por enfermedades transmisibles, la creciente contribución de las enfermedades físicas crónicas y los trastornos mentales a los años vividos con discapacidad han posicionado a estas condiciones entre las principales causas de carga de enfermedad global, representando un serio desafío a los sistemas sanitarios ${ }^{1}$.

En Chile, las enfermedades no transmisibles son responsables de $83,9 \%$ del total de los años de vida ajustados por discapacidad (AVISA), entre estas destacan el subgrupo de las condiciones neu- 
ropsiquiátricas, las enfermedades digestivas y las cardiovasculares ${ }^{2}$. La depresión, posicionándose como la segunda causa de AVISA, ha adquirido tanta relevancia como las enfermedades cardiovasculares para la salud pública chilena ${ }^{2}$.

$\mathrm{Al}$ respecto, la Encuesta Nacional de Salud del año 2009-2010 estimó que 45,3\% de la población general tenía un riesgo cardiovascular considerable, en $24,5 \%$ se sospechaba síntomas respiratorios crónicos, 17,2\% había padecido de síntomas depresivos en el último año, y la presencia de diabetes mellitus fue reportada en $9,4 \%{ }^{3}$.

Complementariamente, los datos de la Encuesta Mundial de Salud de la Organización Mundial de la Salud (OMS), sugieren que, en la población mundial, la prevalencia de un episodio depresivo en personas con enfermedades crónicas (desde $9,3 \%$ para la diabetes mellitus, hasta $23 \%$ para multimorbilidad) es significativamente mayor que en aquellas sin enfermedades crónicas $(3,2 \%)^{4}$.

Se han reportado síntomas elevados de depresión asociados a la presencia de enfermedades crónicas en personas de distintos grupos etarios y procedencia (comunidad ${ }^{5-6}$, atención primaria o ambulatoria ${ }^{7}$, y centros de especialidades $)^{8}$. El hecho que en pacientes con múltiples comorbilidades la enfermedad más común sea la depresión ${ }^{9}$ y que en los depresivos crónicos sea usual la presencia de enfermedades crónicas ${ }^{10}$, retrata la compleja interacción entre estas patologías ${ }^{4}$.

La complejidad de las enfermedades no transmisibles ha llevado al desarrollo de programas de prevención de factores de riesgo y promoción de estilos de vida saludable en la atención primaria de la salud (APS) chilena ${ }^{11}$. No obstante, el abordaje de los trastornos mentales en el escenario de múltiples comorbilidades aún no ha recibido suficiente atención en el país. A pesar de la relevancia clínica y de salud pública que dicho tópico representa para Chile, se trata de un tema poco estudiado, en el que la atención primaria de la salud (APS) deberá jugar un rol importante ${ }^{12}$.

El objetivo de este estudio es caracterizar a personas deprimidas que consultan en centros de APS, considerando la presencia de comorbilidad médica crónica y psiquiátrica.

\section{Material y Método}

Se llevó a cabo un análisis secundario de los datos del diagnóstico basal de un ensayo clínico aleatorio, que tuvo por objetivo evaluar la efectividad de un entrenamiento y supervisión asistidos tecnológicamente para mejorar el manejo de la depresión en equipos de salud de APS en Santiago, Chile ${ }^{13}$.

El marco muestral estuvo compuesto por los centros de salud familiar (CESFAM) de APS en comunas urbanas de Santiago, Región Metropolitana. Se excluyeron a comunas que tuvieran un alto índice de desarrollo humano, alto porcentaje de inmigrantes y adultos mayores, y que dispusieran de psiquiatras en formación trabajando en APS. Se empleó una estrategia de muestreo aleatorio simple en dos pasos para la selección de los establecimientos de salud participantes. En primer lugar, se seleccionaron dos comunas urbanas, y luego, en cada una de estas comunas, se seleccionaron aleatoriamente dos CESFAM, para un total de cuatro establecimientos de salud.

Los equipos de salud de los establecimientos participantes derivaban a personas de ambos sexos, de 18 años o más, en las que se había detectado una probable depresión, según criterio clínico. Un evaluador ciego entrenado era informado de los posibles casos y los contactaba telefónicamente para informar del estudio, consultar por disposición a participar, y acordar fecha para aplicar consentimiento informado, evaluar criterios de inclusión/exclusión, y recabar datos con batería de instrumentos del estudio.

Los criterios de inclusión eran tener 18 años o más, tener un episodio depresivo mayor según la entrevista psiquiátrica estructurada Mini International Neuropsychiatric Interview (MINI) versión Plus 5.0.0 $0^{14}$, y dar consentimiento informado para participar voluntariamente del estudio. Se excluyeron a aquellos que no tenían acceso a teléfono.

La batería de instrumentos aplicada por el evaluador recabó, entre otras variables, antecedentes sociodemográficos, sintomatología depresiva con el Patient Health Questionnaire-9 item (PHQ-9) ${ }^{15}$, morbilidad psiquiátrica (episodio depresivo mayor, riesgo de suicidio, episodio [hipo]maníaco, dependencia de alcohol, trastornos asociados al uso de sustancias psicoactivas no alcohólicas y trastorno de ansiedad generalizada) con la entrevista psiquiátrica estructurada MINI Plus 5.0.0 ${ }^{14}$, y autoreporte de enfermedades crónicas.

El PHQ-9 es un instrumento autoadministrado de nueve ítems, diseñado para evaluar la gravedad de síntomas depresivos y para establecer un diag- 
nóstico provisional de trastorno depresivo ${ }^{15}$. Se trata de un instrumento ampliamente utilizado ${ }^{16}$, cuyo rango de puntuación es de 0-27 puntos. Un puntaje $\geq 10$ indicaría un síndrome depresivo mayor, en tanto que las medidas de gravedad serían: ninguna/mínima (0-4 puntos), leve (5-9 puntos), moderada (10-14 puntos), moderada grave (15-19 puntos), y grave (20-27 puntos $)^{17}$. Ha sido validado en la población chilena ${ }^{18}$.

La entrevista psiquiátrica estructurada MINI Plus 5.0.0 es un breve instrumento de diagnóstico basado en los criterios estandarizados de la cuarta edición del "Manual diagnóstico y estadístico de los trastornos mentales" (DSM-IV), fueron empleados los módulos para diagnosticar episodio depresivo mayor, riesgo de suicidio, episodio (hipo)maníaco, dependencia del alcohol, trastornos asociados al uso de sustancias psicoactivas no alcohólicas y trastorno de ansiedad generalizada ${ }^{14}$. Cuenta con versión en español ${ }^{19}$.

Se realizaron análisis descriptivos de todas las variables, considerándose porcentajes para variables categóricas u ordinales y medias o medianas para variables continuas, dependiendo de la distribución. Se usó la técnica de bootstrap para obtener intervalos de confianza más robustos para las estimaciones, se recurrió a un generador de números aleatorios previamente especificados para asegurar su replicación ${ }^{20}$. Se utilizaron 1.000 replicaciones para permitir el cálculo del intervalo de confianza de sesgo corregido y acelerado (BCa), libre de supuestos de normalidad, proveyendo mayor precisión (20). Los análisis estadísticos fueron asistidos por Stata $12.0^{21}$.

El estudio fue aprobado por el Comité de Ética de Investigación en Seres Humanos de la Facultad de Medicina de la Universidad de Chile (proyecto $\left.n^{\circ} 103-2012\right)$. Se obtuvo el consentimiento informado de todos los participantes.

\section{Resultados}

La muestra estuvo constituida por 256 personas que asistían a cuatro centros de APS en Santiago, Región Metropolitana. Todos ellos presentaban un episodio depresivo según MINI.

Las características socio-demográficas de la muestra se describen en la Tabla 1. La mayoría de las personas eran mujeres, solteras, que habían cursado educación media, con trabajo remunerado y cuya previsión de salud era FONASA A o B. La muestra tenía una mediana de 45,5 años (rango intercuartil 25,5).

Tabla 1. Características socio-demográficas de la muestra de personas deprimidas

\begin{tabular}{|c|c|c|c|}
\hline Variable & & $\begin{array}{c}\text { Total muestra } \\
n=256(\%)\end{array}$ & IC $95 \%{ }^{a}$ \\
\hline Sexo & $\begin{array}{l}\text { Mujer } \\
\text { Hombre }\end{array}$ & $\begin{array}{l}86,72 \\
13,28\end{array}$ & $\begin{array}{c}82,81-90,63 \\
9,77-17,97\end{array}$ \\
\hline Edad (años) & & $45,5^{b}$ & $41-49$ \\
\hline Estado civil & $\begin{array}{l}\text { Casado } \\
\text { Divorciado/Anulado } \\
\text { Soltero } \\
\text { Viudo }\end{array}$ & $\begin{array}{l}35,16 \\
12,89 \\
45,7 \\
6,25\end{array}$ & $\begin{array}{c}30,08-42,58 \\
9,38-18,58 \\
39,06-52,73 \\
3,91-9,77\end{array}$ \\
\hline Nivel educacional & $\begin{array}{l}\text { Básica completa/incompleta } \\
\text { Media completa/incompleta } \\
\text { Técnica profesional completa/incompleta } \\
\text { Universitaria completa/incompleta }\end{array}$ & $\begin{array}{l}17,97 \\
49,61 \\
19,53 \\
12,89\end{array}$ & $\begin{array}{c}14,06-23,44 \\
43,36-56,25 \\
14,84-25,39 \\
9,77-17,97\end{array}$ \\
\hline Caracterización económica & $\begin{array}{l}\text { Ocupados } \\
\text { Desocupados } \\
\text { Inactivos }\end{array}$ & $\begin{array}{c}59,38 \\
3,91 \\
36,72\end{array}$ & $\begin{array}{c}53,91-65,63 \\
2,34-7,03 \\
31,64-42,97\end{array}$ \\
\hline Previsión de salud & $\begin{array}{l}\text { FONASA A/B } \\
\text { FONASA C/D } \\
\text { Otra }\end{array}$ & $\begin{array}{c}65,88 \\
29,41 \\
4,71\end{array}$ & $\begin{array}{c}60,00-70,98 \\
23,14-34,51 \\
2,35-7,45\end{array}$ \\
\hline
\end{tabular}

antervalo de Confianza BCa; ${ }^{\text {'Mediana. }}$ 
Tabla 2. Características clínicas de la muestra de personas deprimidas

\begin{tabular}{|c|c|c|c|}
\hline \multicolumn{2}{|l|}{ Variable } & $\begin{array}{c}\text { Total muestra } \\
n=256(\%)\end{array}$ & IC $95 \% a$ \\
\hline \multicolumn{2}{|c|}{ En tratamiento para episodio depresivo actual } & 27,73 & $23,05-33,98$ \\
\hline \multicolumn{2}{|l|}{ Historia de depresión } & 69,92 & $64,45-75,39$ \\
\hline \multicolumn{2}{|c|}{ Número de episodios depresivos previos } & $2^{b}$ & $1-2^{c}$ \\
\hline Sintomatología depresiva, PHQ-9 & $\begin{array}{l}\text { Ninguna } \\
\text { Leve } \\
\text { Moderada } \\
\text { Moderada grave } \\
\text { Grave }\end{array}$ & $\begin{array}{c}1,60 \\
5,60 \\
26,00 \\
34,40 \\
32,40\end{array}$ & $\begin{array}{c}0,04-3,20 \\
3,20-8,40 \\
21,20-32,40 \\
28,00-40,40 \\
26,40-38,00\end{array}$ \\
\hline Riesgo suicida, MINI & $\begin{array}{l}\text { Ausente } \\
\text { Leve } \\
\text { Moderado } \\
\text { Alto }\end{array}$ & $\begin{array}{c}23,44 \\
37,11 \\
9,38 \\
30,08\end{array}$ & $\begin{array}{c}18,75-29,69 \\
32,03-43,75 \\
6,25-13,67 \\
25,00-35,94\end{array}$ \\
\hline Comorbilidad & $\begin{array}{l}\text { Ninguna } \\
1 \text { o más }\end{array}$ & $\begin{array}{l}21,88 \\
78,13\end{array}$ & $\begin{array}{l}17,19-27,73 \\
73,05-83,59\end{array}$ \\
\hline Tipo de comorbilidad ${ }^{d}$ & $\begin{array}{l}\text { Médica crónicae } \\
\text { Psiquiátrica }^{\dagger} \\
\text { Médica crónica y psiquiátrica }\end{array}$ & $\begin{array}{l}29,00 \\
46,00 \\
25,00\end{array}$ & $\begin{array}{l}23,50-36,50 \\
39,00-53,00 \\
19,50-32,00\end{array}$ \\
\hline
\end{tabular}

alntervalo de Confianza BCa; ${ }^{\text {Mediana; }}$ Intervalo de confianza Percentil; ${ }^{d}$ Categorías excluyentes, el denominador corresponde a los casos que tienen una o más comorbilidades $(n=200) ;{ }^{e}$ Autoreporte; ${ }^{\mathrm{M}} \mathrm{MINI}$.

En la Tabla 2 se resumen las características clínicas de la muestra. Casi tres cuartos de las personas evaluadas referían haber tenido un cuadro depresivo previo, con una mediana de 2 episodios depresivos en el pasado (rango intercuartil 2). De acuerdo al PHQ-9, hubo una mayor proporción de sujetos con cuadros depresivos que calificaron en los rangos moderado grave y grave. Según el MINI, un tercio de los participantes tenía un alto riesgo suicida. Se estableció la presencia de un cuadro depresivo con comorbilidad física, psiquiátrica o ambas en $78,13 \%$ de la muestra.

El $55,47 \%$ de la muestra total padecía alguna comorbilidad psiquiátrica (IC $95 \% \mathrm{BCa}$ $50,00-61,72)$ y la comorbilidad médica crónica fue autoreportada en $42,19 \%$ de todos los casos (IC 95\% BCa 36,72-49,22). Las comorbilidades psiquiátricas más prevalentes fueron el trastorno de ansiedad generalizado $(50,00 \%$, IC $95 \%$ BCa $44,53-56,64$ ) y el episodio (hipo)maníaco $(10,16 \%$, IC $95 \%$ BCa 7,03-14,84), mientras que las enfermedades físicas crónicas más frecuentes fueron hipertensión (20,70\%, IC 95\% BCa 16,02$26,56)$ y diabetes mellitus $(11,72 \%$, IC $95 \% \mathrm{BCa}$ $8,60-16,41)$.

\section{Discusión}

Este estudio se llevó a cabo en centros de APS urbanos y municipales que proveen cuidados continuos e integrales, cercanos a la comunidad, en población que se supone de baja complejidad. En la literatura nacional que aborda la comorbilidad de problemas de salud físicos y mentales, el presente estudio se trata de una de las evidencias más recientes sobre una muestra de un tamaño considerable y que pone énfasis en una de las patologías psiquiátricas que ha alcanzado estatus de problema de salud pública a nivel global, la depresión.

Los resultados sugieren que los consultantes deprimidos en la APS corresponden a una población especialmente compleja. Se trata de casos con historia previa de depresión y episodios depresivos recurrentes. La gravedad del cuadro actual es clara al evaluar la intensidad de la sintomatología depresiva y el riesgo suicida elevado en un porcentaje importante de la muestra. Más aun, la presencia de comorbilidad física o psiquiátrica en más de $70 \%$ de los sujetos retrata la intrincada relación entre estas condiciones. 
La proporción de casos con antecedentes claros de depresión es elevada si se compara con estudios nacionales del mismo grupo poblacional ${ }^{22-24}$. En la literatura internacional, la historia de depresión se ha asociado con una mayor recurrencia del cuadro $^{25}$, observándose que la patología tiene un desarrollo crónico en un tercio de los pacientes deprimidos del nivel primario ${ }^{26-28}$, incrementando el riesgo de padecer un peor estado de salud y discapacidad funcional por períodos prolongados ${ }^{25,29}$.

En la muestra analizada, se evidenciaron niveles moderados a graves de síntomas depresivos, replicando hallazgos previos ${ }^{22-24,30}$. También se detectó una frecuencia de riesgo suicida superior a $70 \%$, cifra cercana a la reportada en hospitales comunitarios de la $\mathrm{X}$ región ${ }^{24}$, pero más alta que la señalada en el extranjero ${ }^{31-32}$. Estos indicadores de compromiso del cuadro clínico son predictores de mala respuesta y recurrencia/cronicidad ${ }^{27,28,33,34}, \mathrm{y}$ parecieran influirse recíprocamente en pacientes depresivos de APS $^{31,32}$.

La comorbilidad de la depresión con enfermedades físicas crónicas y otros trastornos psiquiátricos demostró ser la norma en este estudio, solo cerca de un quinto de los participantes fueron diagnosticados con un episodio depresivo "puro". Estos resultados apuntan en la misma dirección que estudios internacionales que confirmaron la elevada prevalencia de múltiples comorbilidades físicas y mentales en consultantes de APS ${ }^{35-37}$, subrayando la necesidad de dejar atrás la atención de patologías específicas ${ }^{35}$, y relevando el uso de estrategias para la prevención de factores de riesgo comunes ${ }^{36}$.

La relación bidireccional entre la depresión y la hipertensión arterial o la diabetes mellitus se encuentra bien documentada ${ }^{38}$, complicando el pronóstico ${ }^{39,40}$, implicando un mayor uso de servicios $^{41}$, y un aumento en el riesgo de recurrencia del trastorno afectivo ${ }^{42}$. En Chile, los síntomas depresivos en pacientes hipertensos de APS son comunes $^{43}$, y han sido asociados a control insatisfactorio de la presión arterial ${ }^{44}$. Por otra parte, en el extranjero, la prevalencia de trastornos de ansiedad en pacientes deprimidos es frecuente en la $\mathrm{APS}^{45,46}$, encontrándose involucrada en una peor respuesta a tratamiento ${ }^{34}$.

La transición epidemiológica acelerada en Chile $^{47}$ alinea al país con la tendencia global hacia el envejecimiento, y la mayor prevalencia de enfermedades físicas crónicas y problemas de salud mental, con el consecuente aumento de personas con deterioro clínico acumulado que demandarán de servicios de salud oportunos, integrales y de calidad $^{1}$. Este fenómeno se constituye en un llamado urgente a la integración efectiva de la salud física y la salud mental desde la APS, los datos aportados por este estudio son un caso ejemplar.

En cuanto al programa nacional de depresión, la evidencia ha señalado que los equipos de salud de APS adolecen de problemas para detectar el trastorno depresivo ${ }^{48}$ y determinar su gravedad, lo que tiene implicancias al momento de proveer el tratamiento en forma escalonada ${ }^{49}$. Estas inconsistencias acarrean un mayor riesgo de no respuesta a tratamiento, curso recurrente o crónico del cuadro clínico, y mayor discapacidad funcional, contribuyendo conjuntamente a la magnitud de la depresión como problema de salud pública.

No obstante, lo anterior, estas observaciones sobre el manejo de la depresión en la APS han sido realizadas sin considerar la presencia de múltiples comorbilidades sugeridas por este estudio, suponiendo un escenario problemático para equipos de salud que cuentan con guías clínicas que refuerzan un modelo de atención enfocado en patologías específicas ${ }^{50}$. En la misma línea, síntesis de estudios cualitativos sobre barreras para el manejo de la depresión en APS subrayan cómo la presentación enmascarada de la depresión supone una dificultad adicional en contextos de tiempo y recursos acotados ${ }^{51-53}$.

Un denominador común de la muestra estudiada, a la luz de la evidencia revisada, es el potencial impacto negativo de las características clínicas en las posibilidades de remisión. La literatura internacional ha señalado que la reducción del tiempo sin tratamiento en pacientes que inician un nuevo episodio depresivo es clave, sobre todo en el caso del primer episodio depresivo ${ }^{54,55}$. Esto tiene implicancias relevantes para Chile, en donde se ha constatado que la brecha de tratamiento para la depresión en adolescentes es cercana a $90 \%{ }^{56}$.

Este estudio tiene limitaciones para la generalización de sus resultados. En primer lugar, se trata de un análisis secundario de bases de datos de un ensayo clínico aleatorio, lo que pudiera introducir reparos sobre la selección de sujetos que no son representativos de la población deprimida en APS. No obstante, el único criterio de exclusión fueron aquellos sujetos que no contaban con contacto telefónico para la evaluación inicial, sin imponer 
restricciones según alguna característica clínica específica. Un segundo punto que observamos es que se trata de un estudio transversal, que además no incluye un grupo de comparación (sujetos no deprimidos), lo que no nos permite efectuar inferencias válidas respecto del impacto o dirección del efecto en las variables estudiadas. Otra limitación importante es que los participantes fueron derivados por los equipos de salud de APS, lo que pudiera introducir un sesgo de selección, resultando en una muestra de sujetos deprimidos de mayor gravedad y comorbilidad. Sin embargo, aun cuando el sesgo fuese de una magnitud suficiente para introducir diferencias sustantivas, es muy probable que la proporción de sujetos deprimidos con condiciones comórbidas continuara siendo preocupante. Adicionalmente, el autoreporte de enfermedades físicas crónicas en la muestra pudo haber introducido imprecisiones en la prevalencia señalada en este estudio, aun cuando dicha fuente de información ha probado ser válida para un grupo importante de enfermedades crónicas en el contexto de la investigación de múltiples comorbilidades $^{57}$. Finalmente, las comparaciones hechas con la literatura nacional en la sección de discusión pudieran ser artificiosas debido a la diversidad metodológica de los estudios referidos -la mayoría de los datos citados corresponden a la evaluación basal en ensayos clínicos aleatorios. Consideramos que esta es una limitación propia de la producción de evidencia chilena sobre depresión en la APS, estudios que incorporen mediciones de múltiples comorbilidades físicas y psiquiátricas, así como el riesgo de suicidio, son necesarias.

Agradecimientos: Este estudio recibió el apoyo del Fondo de Innovación para la Competitividad (FIC) del Ministerio de Economía, Fomento y Turismo, a través de la Iniciativa Científica Milenio, Proyecto IS130005. Los autores agradecen a los departamentos de salud de las corporaciones municipales, centros de salud de atención primaria y usuarios que participaron de este estudio.

\section{Referencias}

1. Vos T, Barber RM, Bell B, Bertozzi-Villa A, Biryukov S, Bolliger I, et al. Global, regional, and national incidence, prevalence, and years lived with disability for 301 acute and chronic diseases and injuries in 188 countries, 1990-
2013: a systematic analysis for the Global Burden of Disease Study 2013. Lancet 2015; 386 (9995): 743-800.

2. Ministerio de Salud (Chile). Informe Final: Estudio de carga de enfermedad y carga atribuible, Chile 2007. Santiago: Ministerio de Salud (Chile); 2008.

3. Ministerio de Salud (Chile). Encuesta Nacional de Salud ENS Chile 2009-2010. Santiago: Ministerio de Salud (Chile); 2011.

4. Moussavi S, Chatterji S, Verdes E, Tandon A, Patel V, Ustun B. Depression, chronic diseases, and decrements in health: results from the World Health Surveys. Lancet 2007; 370 (9590): 851-88.

5. Kilzieh N, Rastam S, Maziak W, Ward KD. Comorbidity of depression with chronic diseases: a population-based study in Aleppo, Syria. Int J Psychiatry Med 2008; 38 (2): 169-84.

6. De Jonge P, Roy JF, Saz P, Marcos G, Lobo A; ZARADEMP Investigators. Prevalent and incident depression in community-dwelling elderly persons with diabetes mellitus: results from the ZARADEMP project. Diabetologia 2006; 49 (11): 2627-33.

7. Andriopoulos P, Lotti-Lykousa M, Pappa E, Papadopoulos AA, Niakas D. Depression, quality of life and primary care: a cross-sectional study. J Epidemiol Glob Health 2013; 3 (4): 245-52.

8. Quittner AL, Goldbeck L, Abbott J, Duff A, Lambrecht P, Solé A, et al. Prevalence of depression and anxiety in patients with cystic fibrosis and parent caregivers: results of The International Depression Epidemiological Study across nine countries. Thorax 2014; 69 (12): 1090-7.

9. Sinnige J, Braspenning J, Schellevis F, Stirbu-Wagner I, Westert G, Korevaar J. The Prevalence of Disease Clusters in Older Adults with Multiple Chronic Diseases-A Systematic Literature Review. PLoS ONE 2013; 8 (11): e79641.

10. Rubio JM, Markowitz JC, Alegría A, Pérez-Fuentes G, Liu SM, Lin KH, et al. Epidemiology of chronic and nonchronic major depressive disorder: results from the national epidemiologic survey on alcohol and related conditions. Depress Anxiety 2011; 28 (8): 622-31.

11. Ministerio de Salud (Chile). Estrategia Nacional de Salud para el Cumplimiento de los Objetivos Sanitarios de la Década 2011-2020. Santiago: MINSAL; 2011.

12. Fullerton C, Florenzano R, Acuña J. Comorbilidad de enfermedades médicas crónicas y trastornos psiquiátricos en una población de consultantes en el nivel primario de atención. Rev Med Chile 2000; 128 (7): 729-34.

13. Rojas G, Martínez P, Vöhringer PA, Martínez V, Castro-Lara A, Fritsch R. Comprehensive technology-assisted training and supervision program to enhance depression management in primary care in Santiago, 
Chile: study protocol for a cluster randomized controlled trial. Trials 2015; 16: 311.

14. Sheehan DV, Lecrubier Y, Harnett F, Sheehan K, Amorim P, Janavs J, et al. The Mini International Neuropsychiatric Interview (M.I.N.I.): The development and validation of a structured diagnostic psychiatric interview. J Clin Psychiatry 1998; 59 (Suppl 20): 22-33.

15. Spitzer RL, Kroenke K, Williams JBW. Validation and utility of a self-report version of PRIME-MD: the PHQ primary care study. JAMA 1999; 282 (18): 1737-44.

16. Kroenke K, Spitzer RL, Williams JB, Lowe B. The patient health questionnaire somatic, anxiety, and depressive symptom scales: a systematic review. Gen Hosp Psychiatry 2010; 32 (4): 345-59.

17. Kroenke K, Spitzer RL, Williams JB. The PHQ-9: validity of a brief depression severity measure. J Gen Intern Med 2001; 16 (9): 606-13.

18. Baader T, Molina JL, Venezian S, Rojas C, Farías R, Fierro-Freixenet C, y cols. Validación y utilidad de la encuesta PHQ-9 (Patient Health Questionnaire) en el diagnóstico de depresión en pacientes usuarios de atención primaria. Rev Chil Neuro-Psiquiat 2012; 50 (1): 10-22.

19. Ferrando L, Bobes J, Gibert J, Lecrubier Y. Mini International Neuropsychiatric Interview. En: Bobes J, Bousoño M, González MP, editores. Manejo de los trastornos mentales y del comportamiento en Atención Primaria. $2^{\circ}$ ed. Oviedo: Gofer; 1997.

20. Carpenter J, Bithell J. Bootstrap confidence intervals: when, which, what? A practical guide for medical statisticians. Statis Med 2000; 19: 1141-64.

21. StataCorp. Stata Statistical Software: Release 12. College Station, TX: StataCorp LP.

22. Araya R, Rojas G, Fritsch R, Gaete J, Rojas M, Simon G, et al. Treating depression in primary care in low-income women in Santiago, Chile: a randomised controlled trial. Lancet 2003; 361: 995-1000.

23. Fritsch R, Araya R, Solís J, Montt E, Pilowsky D, Rojas G. Un ensayo clínico aleatorizado de farmacoterapia con monitorización telefónica para mejorar el tratamiento de la depresión en la atención primaria en Santiago, Chile. Rev Med Chile 2007; 135 (5): 587-95.

24. Rojas G, Castro A, Guajardo V, Alvarado R, Isamit C, Fritsch R. Programa colaborativo a distancia para el tratamiento de la enfermedad depresiva. Rev Med Chile 2014; 142 (9): 1142-9.

25. Hardeveld F, Spikjer J, De Graaf R, Hendriks SM, Licht CMM, Nolen WA, et al. Recurrence of major depressive disorder across different treatment settings: Results from the NESDA study. J Affect Disorders 2013; 147: 225-31.

26. Interian A, Ang A, Gara MA, Rodríguez MA, Vega WA.
The long-term trajectory of depression among Latinos in primary care and its relationship to depression care disparities. Gen Hosp Psychiatry 2011; 33 (2): 94-101.

27. Riihimäki KA, Vuorilehto MS, Melartin TK, Isometsä ET. Five-year outcome of major depressive disorder in primary health care. Psychol Med 201444 (7): 1369-79.

28. Wardenaar KJ, Conradi HJ, de Jonge P. Data-driven course trajectories in primary care patients with major depressive disorder. Depress Anxiety 2014; 31: 778-86.

29. Riihimäki K, Vuorilehto M, Isometsä E. A 5-year prospective study of predictors for functional and work disability among primary care patients with depressive disorders. Eur Psychiatry 2015; 30 (1): 51-7.

30. Vöhringer PA, Jiménez MI, Igor MA, Forés GA, Correa $\mathrm{MO}$, Sullivan MC et al. A clinical predictive score for mood disorder risk in low-income primary care settings. J Affect Disorders 2013; 151: 1125-31.

31. Moreno-Küstner B, Jones R, Svab I, Maaroos H, Xavier $M$, Geerlings $M$, et al. Suicidality in primary care patients who present with sadness and anhedonia: a prospective European study. BMC Psychiatry 2016; 16: 94.

32. Gensichen K, Teising A, König J, Gerlach FM, Petersen JJ. Predictors of suicidal ideation in depressive primary care patients. J Affect Disorders 2010; 125 (1-3): 124-7.

33. Stegenga BT, Kamphuis MH, King M, Nazareth I, Geerlings MI. The natural course and outcome of major depressive disorder in primary care: the PREDICT-NL study. Soc Psychiatry Psychiatr Epidemiol 2012; 47 (1): 87-95.

34. De Carlo V, Calati R, Serretti A. Socio-demographic and clinical predictors of non-response/non-remission in treatment resistant depressed patients: A systematic review. Psychiatry Res 2016; 240: 421-30.

35. Barnett K, Mercer SW, Norbury M, Watt G, Wyke S, Guthrie B. Epidemiology of multimorbidity and implications for health care, research, and medical education: a cross-sectional study. Lancet 2012; 380: 37-43.

36. Violan C, Foguet-Boreu Q, Flores-Mateo G, Salisbury C, Blom J, Freitag M, et al. Prevalence, Determinants and Patterns of Multimorbidity in Primary Care: A Systematic Review of Osbservational Studies. PLoS One 2014; 9 (7): e102149.

37. Gil M, Comas A, García-García M, Monzón S, Antoni S-B, Roca M. Comorbidity between common mental disorders and chronic somatic diseases in primary care patients. Gen Hosp Psychiatry 2010; 32 (3): 240-5.

38. Katon WJ. Epidemiology and treatment of depression in patients with chronic medical illness. Dialogues Clin Neurosci 2011; 13 (1): 7-23.

39. Cuijpers P, Vogelzangs N, Twisk J, Kleiboer A, Li J, 
Penninx BW. Comprehensive Meta-Analysis of Excess Mortality in Depression in the General Community Versus Patients With Specific Illnesses. Am J Psychiatry 2014; 171: 453-62.

40. Hofmann M, Köhler B, Leichsenring F, Kruse J. Depression as a Risk Factor for Mortality in Individuals with Diabetes: A Meta-Analysis of Prospective Studies. PLoS One 2013; 8 (11): e79809.

41. Dickens C, Katon W, Blakemore A, Khara A, McGowan $\mathrm{L}$, Tomenson B, et al. Does depression predict the use of urgent and unscheduled care by people with long term conditions? A systematic review with meta-analysis. J Psychosom Res 2012; 73: 334-42.

42. Nefs G, Pouwer F, Denollet J, Pop V. The course of depressive symptoms in primary care patients with type 2 diabetes: results from the Diabetes, Depression, Type D Personality Zuidoost-Brabant (DiaDDZoB) Study. Diabetologia 2012; 55 (3): 608-16.

43. Dois A, Cazenave A. Pesquisa de depresión mayor en hipertensos consultantes de la Atención Primaria. Rev Med Chile 2009; 137 (4): 475-80.

44. Garrido J, Chacón J, Sandoval D, Muñoz R, López N, Oyarzún E, et al. Control del Hipertenso, un desafío no resuelto. Avances logrados en Chile mediante el Programa de Salud Cardiovascular. Rev Chil Cardiol 2013; 32 (2): 85-96

45. Olfson M, Shea S, Feder A, Fuentes M, Nomura Y, Gameroff M, et al. Prevalence of Anxiety, Depression, and Substance Use Disorders in an Urban General Medicine Practice. Arch Fam Med 2000; 9: 876-83.

46. Martín-Merino E, Rui Gómez A, Johansson S, Wallander M-A, García-Rodríguez LA. Study of a Cohort of Patients Newly Diagnosed With Depression in General Practice: Prevalence, Incidence, Comorbidity, and Treatment Patterns. Prim Care Companion J Clin Psychiatry 2010; 12 (1): PCC.08m00764.

47. Luque C, Cisternas FA, Araya M. Cambios en el patrón de enfermedad en la postransición epidemiológica en salud en Chile, 1950-2003. Rev Med Chile 2006; 134: 703-12.

48. Vöhringer PA, Jiménez MI, Igor MA, Fores GA, Correa MO, Sullivan MC, et al. Detecting Mood Disorder in
Resource-Limited Primary Care Settings: Comparison of a self-administered screening tool to general practitioner assessment. J Med Screen 2013; 20 (3): 118-24.

49. Alvarado R, Rojas G. El programa nacional para el diagnóstico y tratamiento de depresión en atención primaria: una evaluación necesaria. Rev Med Chile 2011; (139): 592-9.

50. Barnett K, Mercer SW, Norbury M, Watt G, Wyke S, Guthrie B. Epidemiology of multimorbidity and implications for health care, research, and medical education: a cross-sectional study. Lancet 2012; 380 (9836): 37-43.

51. Barley EA, Murray J, Walters P, Tylee A. Managing depression in primary care: A meta-synthesis of qualitative and quantitative research from the UK to identify barriers and facilitators. BMC Fam Pract 2011; 12 (1): 47.

52. Schumann I, Schneider A, Kantert C, Löwe B, Linde K. Physicians' attitudes, diagnostic process and barriers regarding depression diagnosis in primary care: A systematic review of qualitative studies. Fam Pract 2012; 29 (3): 255-63.

53. McPherson S, Armstrong D. General Practitioner Management of Depression: A Systematic Review. Qual Health Res 2012; 22 (8): 1150-9.

54. Ghio L, Gotelli S, Marcenaro M, Amore M, Natta W. Duration of untreated illness and outcomes in unipolar depression: A systematic review and meta-analysis. J Affect Disorders 2014; 152-154: 45-51.

55. Hung CI, Yu NW, Liu CY, Wu KY, Yang CH. The impact of the duration of an untreated episode on improvement of depression and somatic symptoms. Neuropsychiatr Dis Treat 2015; 11: 2245-52.

56. Vicente B, Saldivia S, de la Barra F, Melipillán R, Valdivia M, Kohn R. Salud mental infanto-juvenil en Chile y brechas de atención sanitarias. Rev Med Chile 2012; 140: 447-57.

57. Hansen H, Schäfer I, Schön G, Riedel-Heller S, Gensinchen J, Weyerer S, et al. Agreement between self-reported and general practitioner-reported chronic conditions among multimorbid patients in primary care-results of the MultiCare Cohort Study. BMC Fam Pract 2014; 15: 39. 